\title{
Total Synthesis of Abyssenine A. Application of Inter- and Intramolecular Copper-mediated Coupling Reactions in Organic Synthesis
}

\author{
Mathieu Toumi, François Couty and Gwilherm Evano* \\ Institut Lavoisier de Versailles, UMR CNRS 8180 \\ Université de Versailles Saint-Quentin en Yvelines \\ 45, avenue des Etats-Unis \\ 78035 Versailles Cedex \\ France
}

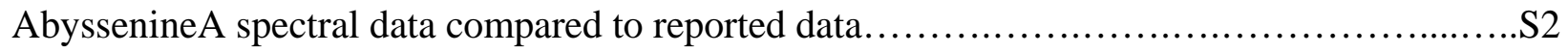

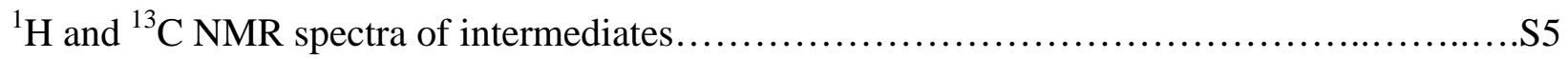

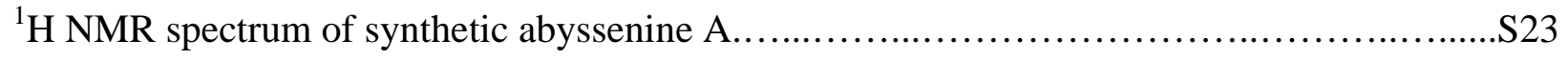

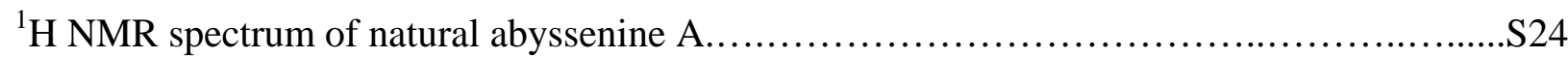

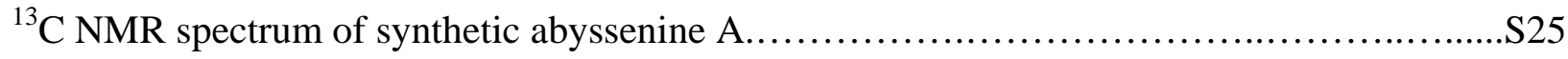

${ }^{1} \mathrm{H}-{ }^{1} \mathrm{H}$ COSY and ${ }^{1} \mathrm{H}^{13}{ }^{13} \mathrm{C}$ HSQC NMR spectra of synthetic abyssenine A.......................S26 


\section{Abyssenine A Spectral Data Compared to Reported Data}

${ }^{1} \mathbf{H}$ NMR (Synthetic at $300 \mathrm{MHz}$; Natural at 90 and $300 \mathrm{MHz}$ )

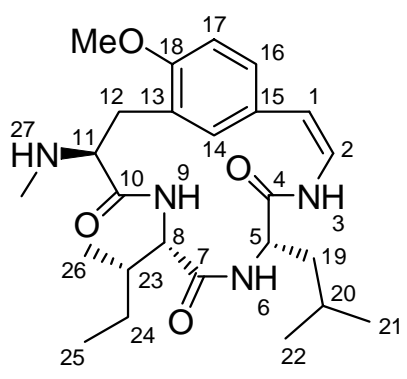

Abyssenine $A$

\begin{tabular}{|c|c|c|c|c|c|c|c|c|}
\hline \multirow[b]{2}{*}{ Position } & \multicolumn{2}{|c|}{ Synthetic (300 MHz) } & \multicolumn{3}{|c|}{ Natural (Tsheche, $90 \mathrm{MHz})^{\mathrm{S1}}$} & \multicolumn{3}{|c|}{ Natural (Bodo, $300 \mathrm{MHz})^{\mathrm{S} 2}$} \\
\hline & $\delta$ & multiplicity & $\delta$ & multiplicity & $\Delta \delta$ & $\delta$ & multiplicity & $\Delta \delta$ \\
\hline $1(1 \mathrm{H})$ & 5.60 & $\mathrm{~d}, J=9.6 \mathrm{~Hz}$ & 5.60 & $\mathrm{~d}, J=9.6 \mathrm{~Hz}$ & 0.00 & 5.58 & $\mathrm{~d}, J=9.6 \mathrm{~Hz}$ & 0.02 \\
\hline $2(1 \mathrm{H})$ & 6.83 & $\mathrm{dd}, J=11.3,9.6 \mathrm{~Hz}$ & 6.72 & $\mathrm{~d}, J=10 \mathrm{~Hz}$ & 0.11 & 6.81 & $\mathrm{dd}, J=11.3,9.6 \mathrm{~Hz}$ & 0.02 \\
\hline $3(1 \mathrm{H})$ & 8.47 & $\mathrm{~d}, J=11.3 \mathrm{~Hz}$ & 8.50 & $\mathrm{~d}, J=12 \mathrm{~Hz}$ & 0.03 & 8.45 & $\mathrm{~d}, J=11.3 \mathrm{~Hz}$ & 0.02 \\
\hline $5(1 \mathrm{H})$ & 4.47 & ddd, $J=11.1,7.7,3.6 \mathrm{~Hz}$ & $\mathrm{NR}^{\mathrm{C}}$ & $\mathrm{NR}^{\mathrm{C}}$ & $\mathrm{NA}^{\mathrm{b}}$ & 4.45 & $\mathrm{~m}, J=7.6,3.5 \mathrm{~Hz}$ & 0.02 \\
\hline $6(1 \mathrm{H})$ & 9.43 & $\mathrm{~d}, J=7.5 \mathrm{~Hz}$ & 9.45 & $\mathrm{~d}, J=8 \mathrm{~Hz}$ & 0.02 & 9.37 & br s & 0.06 \\
\hline $8(1 \mathrm{H})$ & $3.25-3.32$ & $\mathrm{~m}$ & $\mathrm{NR}^{\mathrm{C}}$ & $\mathrm{NR}^{\mathrm{c}}$ & $\mathrm{NA}^{\mathrm{b}}$ & 3.30 & $\mathrm{~m}$ & -0.01 \\
\hline $9(1 \mathrm{H})$ & 8.16 & $\mathrm{~d}, J=7.2 \mathrm{~Hz}$ & 8.15 & $\mathrm{~d}, J=7 \mathrm{~Hz}$ & 0.01 & 8.16 & br $\mathrm{s}$ & 0.00 \\
\hline
\end{tabular}

${ }^{S 1}$ Tschesche, R.; David, S. T.; Zerbes, R.; von Radloff, M.; Kaussmann, E. U.; Eckhardt, G. Liebigs Ann. Chem. 1974, 1915-1928.

${ }^{S 2}$ Auvin, C.; Lezenven, F.; Blond, A.; Augeven-Bour, I.; Pousset, J.-L.; Bodo, B. unpublished results. 


\begin{tabular}{|c|c|c|c|c|c|c|c|c|}
\hline $11(1 \mathrm{H})$ & $3.25-3.32$ & $\mathrm{~m}$ & $\mathrm{NR}^{\mathrm{c}}$ & $\mathrm{NR}^{\mathrm{C}}$ & $\mathrm{NA}^{\mathrm{b}}$ & 3.30 & $\mathrm{~m}$ & -0.01 \\
\hline $12(1 \mathrm{H})$ & 3.46 & $\mathrm{dd}, J=13.6,6.1 \mathrm{~Hz}$ & $\mathrm{NR}^{\mathrm{c}}$ & $\mathrm{NR}^{\mathrm{C}}$ & $\mathrm{NA}^{\mathrm{b}}$ & 3.43 & $\mathrm{dd}, J=13.5,6.0 \mathrm{~Hz}$ & 0.03 \\
\hline $12^{\prime}(1 \mathrm{H})$ & 3.04 & $\mathrm{dd}, J=13.6,2.3 \mathrm{~Hz}$ & $\mathrm{NR}^{\mathrm{C}}$ & $\mathrm{NR}^{\mathrm{c}}$ & $\mathrm{NA}^{\mathrm{b}}$ & 3.03 & $\mathrm{dd}, J=13.5,3.0 \mathrm{~Hz}$ & 0.01 \\
\hline $14(1 \mathrm{H})$ & 7.14 & $\mathrm{~d}, J=2.1 \mathrm{~Hz}$ & $6.70-7.30$ & $\mathrm{~m}$ & $\mathrm{NA}^{\mathrm{b}}$ & 7.12 & $\mathrm{~d}, J=2.1 \mathrm{~Hz}$ & 0.02 \\
\hline $16(1 \mathrm{H})$ & 7.01 & $\mathrm{dd}, J=8.5,2.2 \mathrm{~Hz}$ & $6.70-7.30$ & $\mathrm{~m}$ & $\mathrm{NA}^{\mathrm{b}}$ & 6.99 & $\mathrm{dd}, J=8.5,2.1 \mathrm{~Hz}$ & 0.02 \\
\hline $17(1 \mathrm{H})$ & 6.88 & $\mathrm{~d}, J=8.6 \mathrm{~Hz}$ & $6.70-7.30$ & $\mathrm{~m}$ & $\mathrm{NA}^{\mathrm{b}}$ & 6.86 & $\mathrm{~d}, J=8.5 \mathrm{~Hz}$ & 0.02 \\
\hline $19(1 \mathrm{H})$ & $1.92-2.00$ & $\mathrm{~m}$ & $\mathrm{NR}^{\mathrm{c}}$ & $\mathrm{NR}^{\mathrm{c}}$ & $\mathrm{NA}^{\mathrm{b}}$ & 1.95 & $\mathrm{~m}$ & 0.01 \\
\hline 19’ (1H) & $1.71-1.84$ & $\mathrm{~m}$ & $\mathrm{NR}^{\mathrm{C}}$ & $\mathrm{NR}^{\mathrm{c}}$ & $\mathrm{NA}^{\mathrm{b}}$ & 1.75 & $\mathrm{~m}$ & 0.02 \\
\hline $20(1 \mathrm{H})$ & $1.71-1.84$ & $\mathrm{~m}$ & $\mathrm{NR}^{\mathrm{c}}$ & $\mathrm{NR}^{\mathrm{c}}$ & $\mathrm{NA}^{\mathrm{b}}$ & 1.75 & $\mathrm{~m}$ & 0.02 \\
\hline $21(3 \mathrm{H})$ & $1.02^{\mathrm{a}}$ & $\mathrm{d}, J=6.3 \mathrm{~Hz}$ & $0.70-1.10$ & $\mathrm{~m}$ & $\mathrm{NA}^{\mathrm{b}}$ & $0.99^{\mathrm{a}}$ & $\mathrm{d}, J=6.3 \mathrm{~Hz}$ & 0.03 \\
\hline $22(3 \mathrm{H})$ & $0.96^{\mathrm{a}}$ & $\mathrm{d}, J=6.3 \mathrm{~Hz}$ & $0.70-1.10$ & $\mathrm{~m}$ & $\mathrm{NA}^{\mathrm{b}}$ & $0.93^{\mathrm{a}}$ & $\mathrm{d}, J=6.3 \mathrm{~Hz}$ & 0.03 \\
\hline $23(3 \mathrm{H})$ & $2.47-2.60$ & $\mathrm{~m}$ & $\mathrm{NR}^{\mathrm{c}}$ & $\mathrm{NR}^{\mathrm{c}}$ & $\mathrm{NA}^{\mathrm{b}}$ & 2.5 & $\mathrm{~m}$ & $\mathrm{NA}^{\mathrm{b}}$ \\
\hline $24(1 \mathrm{H})$ & 1.52 & $\mathrm{dqd}, J=10.6,7.5,3.2 \mathrm{~Hz}$ & $\mathrm{NR}^{\mathrm{C}}$ & $\mathrm{NR}^{\mathrm{C}}$ & $\mathrm{NA}^{\mathrm{b}}$ & 1.50 & $\mathrm{~m}$ & 0.02 \\
\hline $24^{\prime}(1 \mathrm{H})$ & 1.14 & $\mathrm{dqd}, J=10.6,7.5,7.5$ & $\mathrm{NR}^{\mathrm{C}}$ & $\mathrm{NR}^{\mathrm{C}}$ & $\mathrm{NA}^{\mathrm{b}}$ & 1.20 & $\mathrm{~m}$ & -0.06 \\
\hline $25(3 \mathrm{H})$ & 0.86 & $\mathrm{t}, J=7.5 \mathrm{~Hz}$ & $0.70-1.10$ & $\mathrm{~m}$ & $\mathrm{NA}^{\mathrm{b}}$ & 0.84 & t, $J=7.4 \mathrm{~Hz}$ & 0.02 \\
\hline $26(3 \mathrm{H})$ & 0.90 & $\mathrm{~d}, J=6.8 \mathrm{~Hz}$ & $0.70-1.10$ & $\mathrm{~m}$ & $\mathrm{NA}^{\mathrm{b}}$ & 0.88 & $\mathrm{~d}, J=6.8 \mathrm{~Hz}$ & 0.02 \\
\hline $27(1 \mathrm{H})$ & 2.18 & br. s & $\mathrm{NR}^{\mathrm{C}}$ & $\mathrm{NR}^{\mathrm{C}}$ & $\mathrm{NA}^{\mathrm{b}}$ & $\mathrm{NR}^{\mathrm{c}}$ & $\mathrm{NR}^{\mathrm{C}}$ & $\mathrm{NA}^{\mathrm{b}}$ \\
\hline OMe (3H) & 3.86 & $\mathrm{~s}$ & 3.85 & $\mathrm{~s}$ & 0.01 & 3.84 & $\mathrm{~s}$ & 0.02 \\
\hline NMe (3H) & 2.48 & $\mathrm{~s}$ & 2.50 & $\mathrm{~s}$ & -0.02 & 2.46 & $\mathrm{~s}$ & 0.02 \\
\hline
\end{tabular}

a both assignments can be interchanged

${ }^{\mathrm{b}} \mathrm{NA}=$ not available

${ }^{\mathrm{c}} \mathrm{NR}=$ not reported 
${ }^{13}$ C NMR (Synthetic at $75 \mathrm{MHz}$; Natural at $75 \mathrm{MHz}$ )

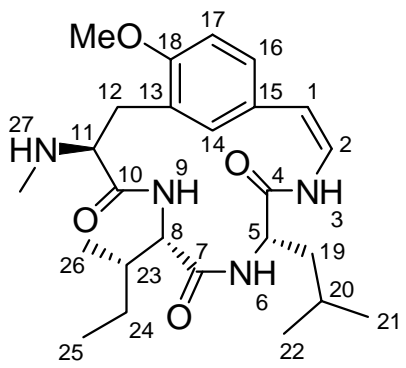

Abyssenine $A$

\begin{tabular}{|c|c|c|c|c|}
\hline & Synthetic & (Bod & & $\begin{array}{c}\text { Natural } \\
\text { (Tsheche) }\end{array}$ \\
\hline Position & $\delta$ & $\delta$ & $\Delta \delta$ & \multirow{7}{*}{$\mathrm{N}$} \\
\hline 1 & 109.8 & 109.7 & 0.1 & \\
\hline 2 & 120.5 & 120.4 & 0.1 & \\
\hline 4 & 170.1 & 170.0 & 0.1 & \\
\hline 5 & 52.5 & 52.5 & 0.0 & \\
\hline 7 & $172.3^{\mathrm{a}}$ & 172.3 & 0.0 & \\
\hline 8 & $65.8^{b}$ & $\mathrm{NR}^{\mathrm{e}}$ & $\mathrm{NA}^{\mathrm{f}}$ & \\
\hline 10 & $176.2^{\mathrm{a}}$ & $\mathrm{NR}^{\mathrm{e}}$ & $\mathrm{NA}^{\mathrm{f}}$ & \multirow{4}{*}{$\begin{array}{l}\mathrm{O} \\
\mathrm{T}\end{array}$} \\
\hline 11 & $66.3^{b}$ & 66.0 & 0.3 & \\
\hline 12 & 31.3 & 31.3 & 0.0 & \\
\hline 13 & $125.2^{\mathrm{c}}$ & 124.9 & 0.3 & \\
\hline 14 & 128.99 & 128.9 & 0.1 & $\mathrm{R}$ \\
\hline 15 & $129.04^{\mathrm{C}}$ & 128.9 & 0.0 & \multirow[t]{2}{*}{ E } \\
\hline 16 & 129.9 & 129.8 & 0.1 & \\
\hline 17 & 111.7 & 111.5 & 0.0 & $\mathrm{P}$ \\
\hline 18 & 156.3 & 156.1 & 0.2 & \multirow{2}{*}{$\mathrm{O}$} \\
\hline 19 & 41.2 & 41.0 & 0.2 & \\
\hline 20 & 25.2 & 25.1 & 0.1 & $\begin{array}{l}\mathrm{R} \\
\mathrm{T}\end{array}$ \\
\hline 21 & $23.5^{d}$ & 23.4 & 0.1 & \multirow{2}{*}{ E } \\
\hline 22 & $21.1^{\mathrm{d}}$ & 21.0 & 0.1 & \\
\hline 23 & 33.4 & 33.5 & -0.1 & \multirow[t]{6}{*}{ D } \\
\hline 24 & 25.9 & 25.7 & 0.2 & \\
\hline 25 & 15.4 & 15.3 & 0.1 & \\
\hline 26 & 9.7 & 9.7 & 0.0 & \\
\hline OMe & 55.8 & 55.7 & 0.1 & \\
\hline NMe & 36.6 & 36.2 & 0.4 & \\
\hline
\end{tabular}

${ }^{a}$ both assignments can be interchanged

${ }^{\mathrm{b}}$ both assignments can be interchanged

${ }^{\mathrm{c}}$ both assignments can be interchanged
${ }^{\mathrm{d}}$ both assignments can be interchanged

${ }^{\mathrm{e}} \mathrm{NR}=$ not reported

${ }^{\mathrm{f}} \mathrm{NA}=$ not available

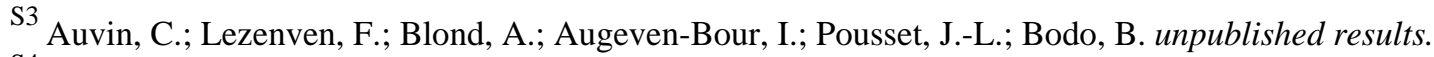

${ }^{S 4}$ Tschesche, R.; David, S. T.; Zerbes, R.; von Radloff, M.; Kaussmann, E. U.; Eckhardt, G. Liebigs Ann. Chem. 1974, 1915-1928. 
Supporting Information

\section{${ }^{1} \mathrm{H}$ and ${ }^{13} \mathrm{C}$ NMR spectra of intermediates and Abyssenine A}




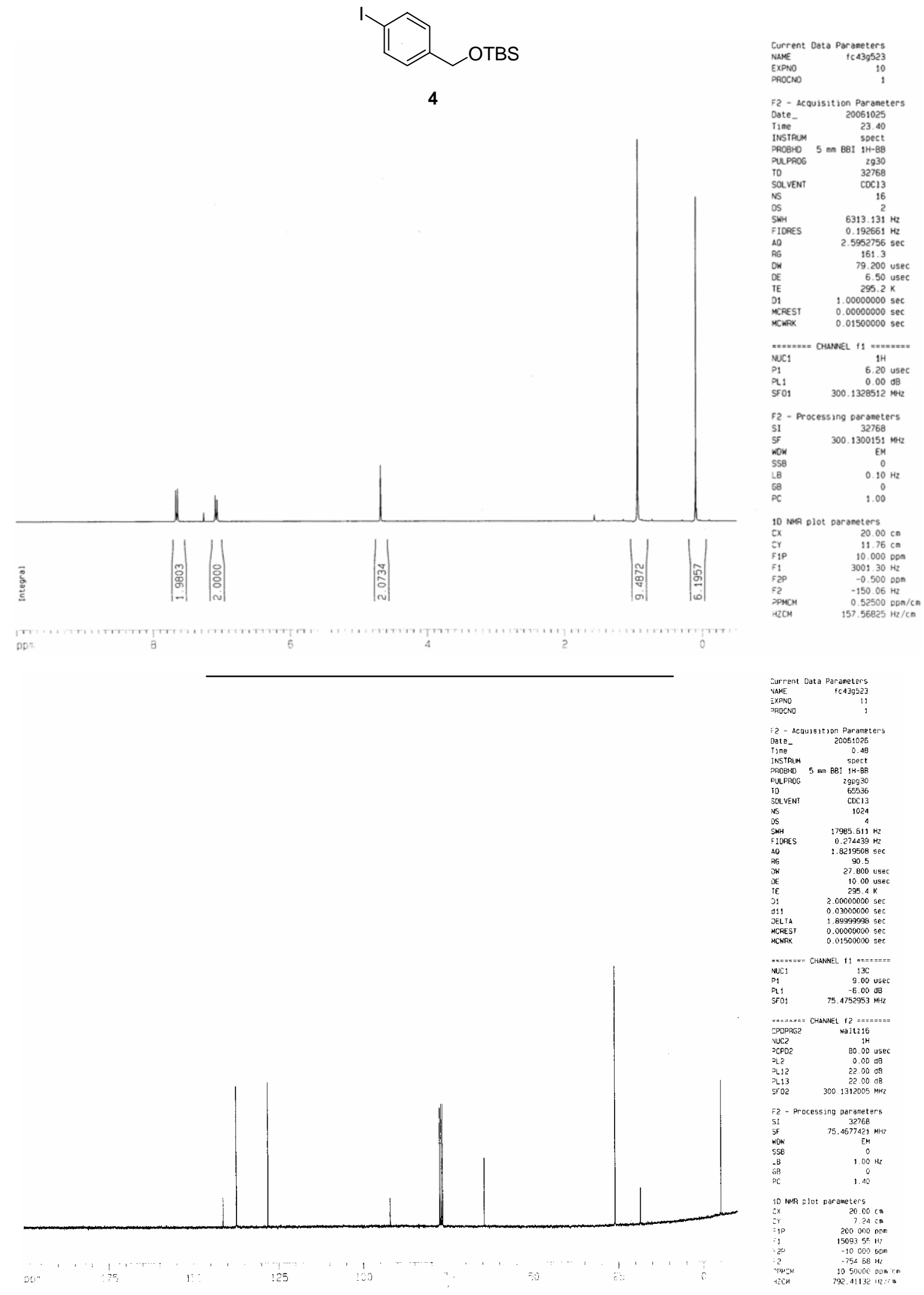




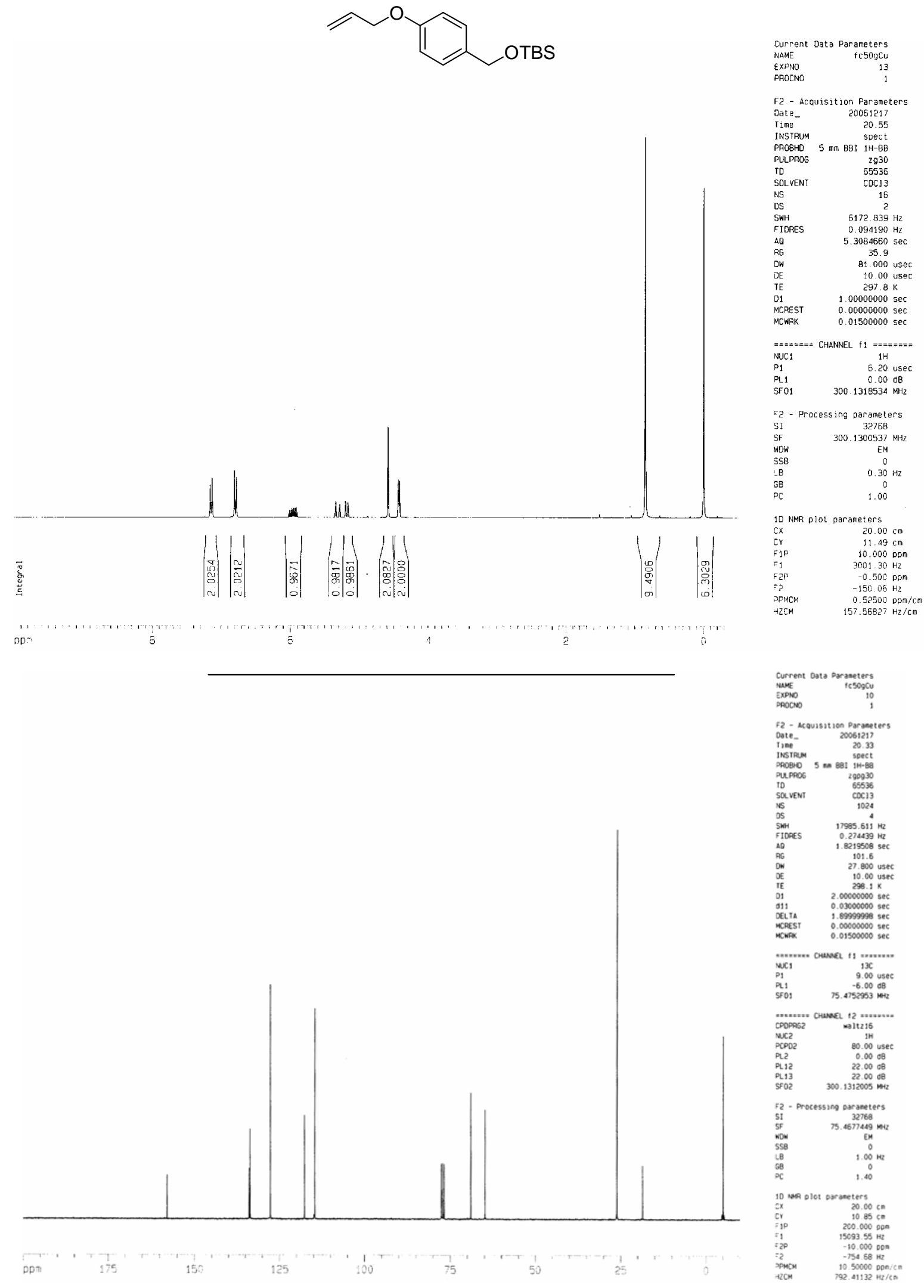




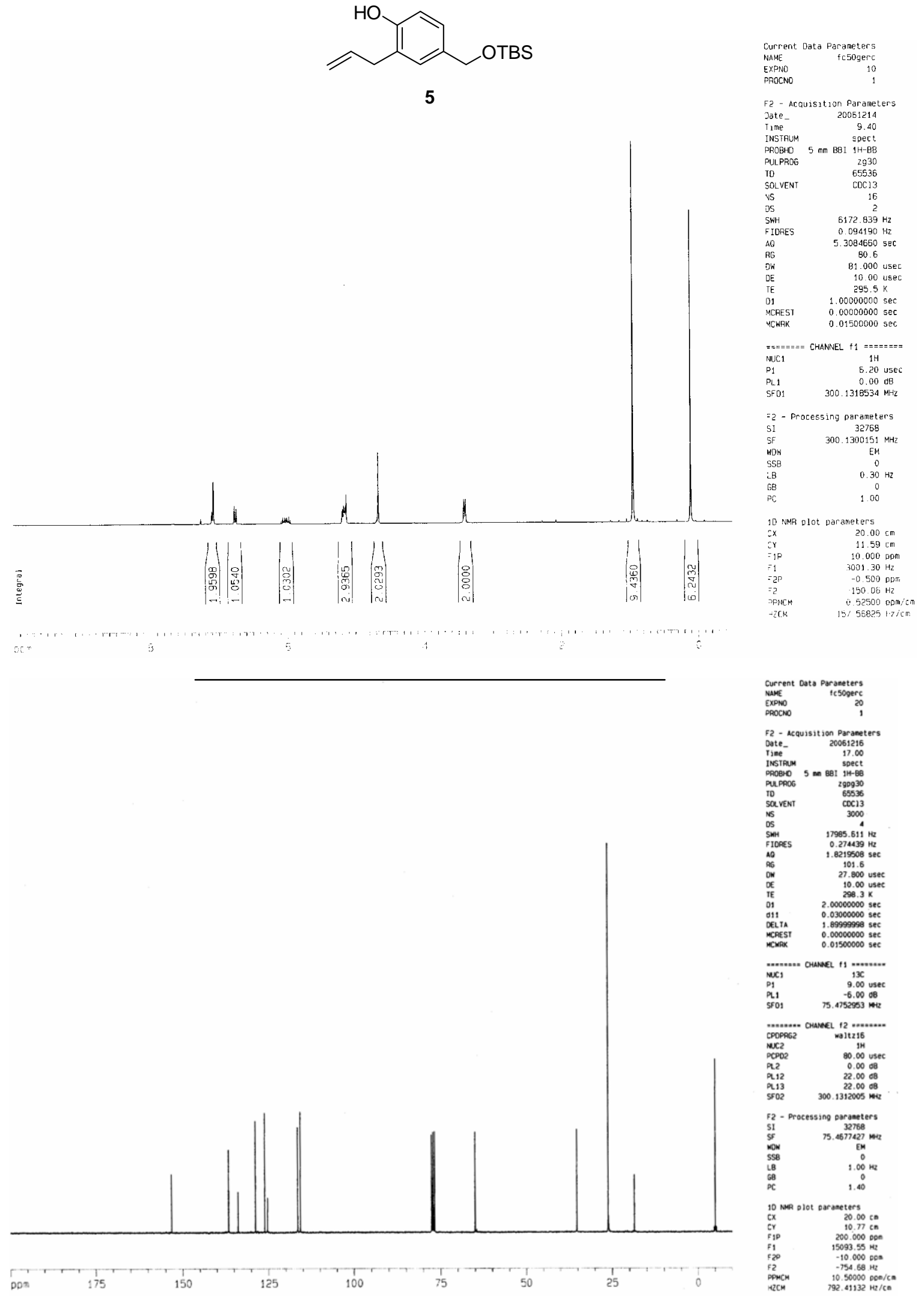




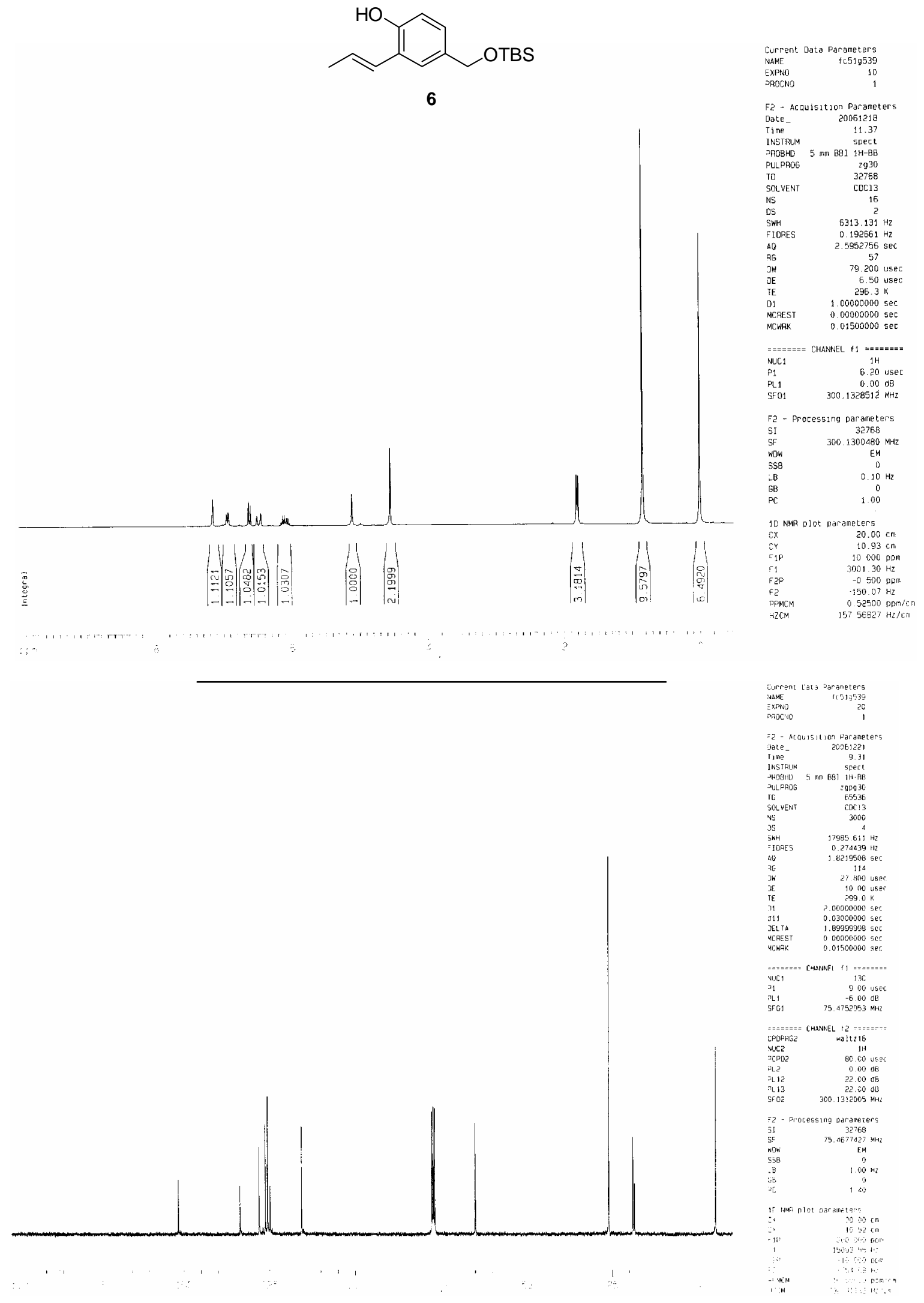




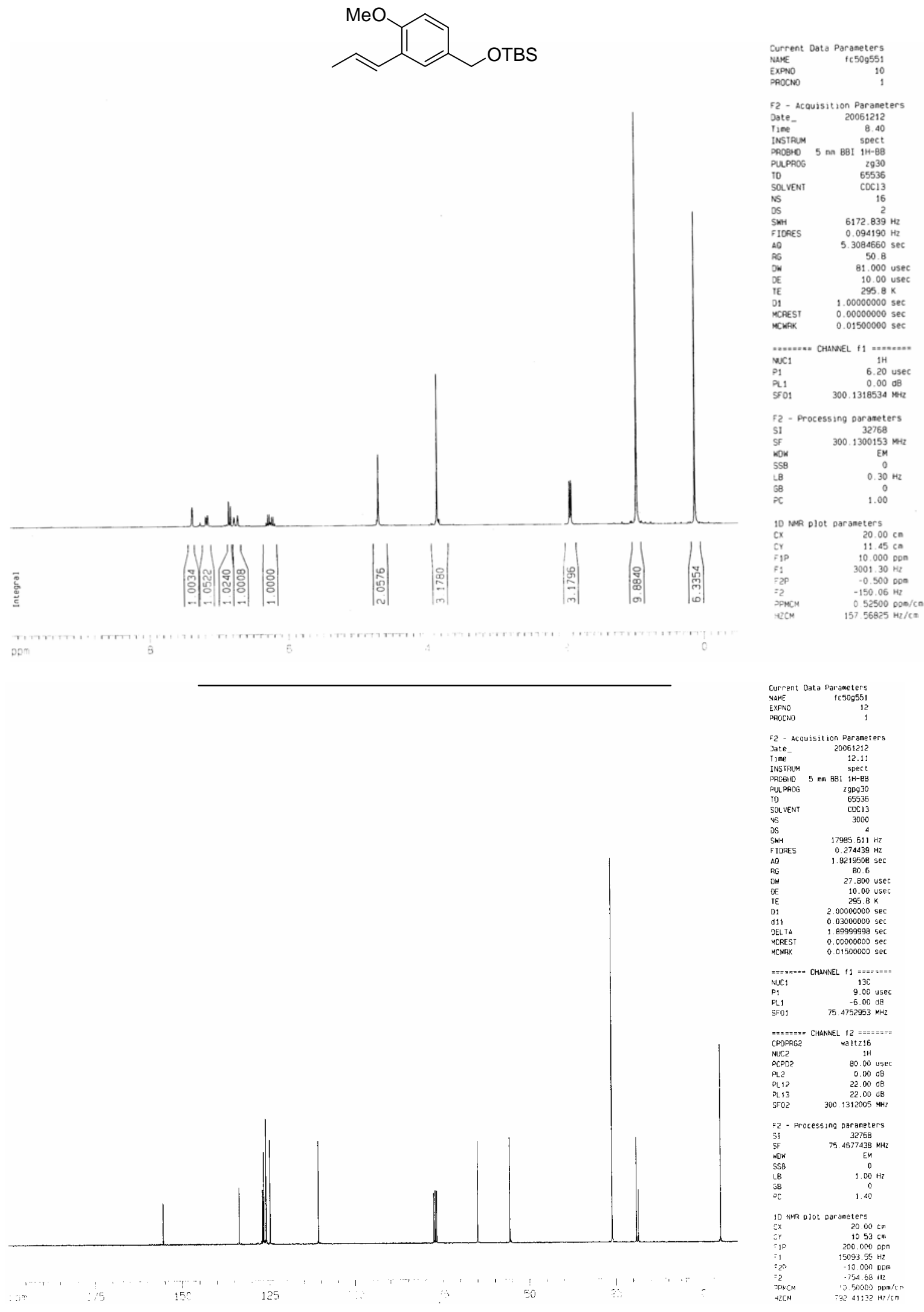




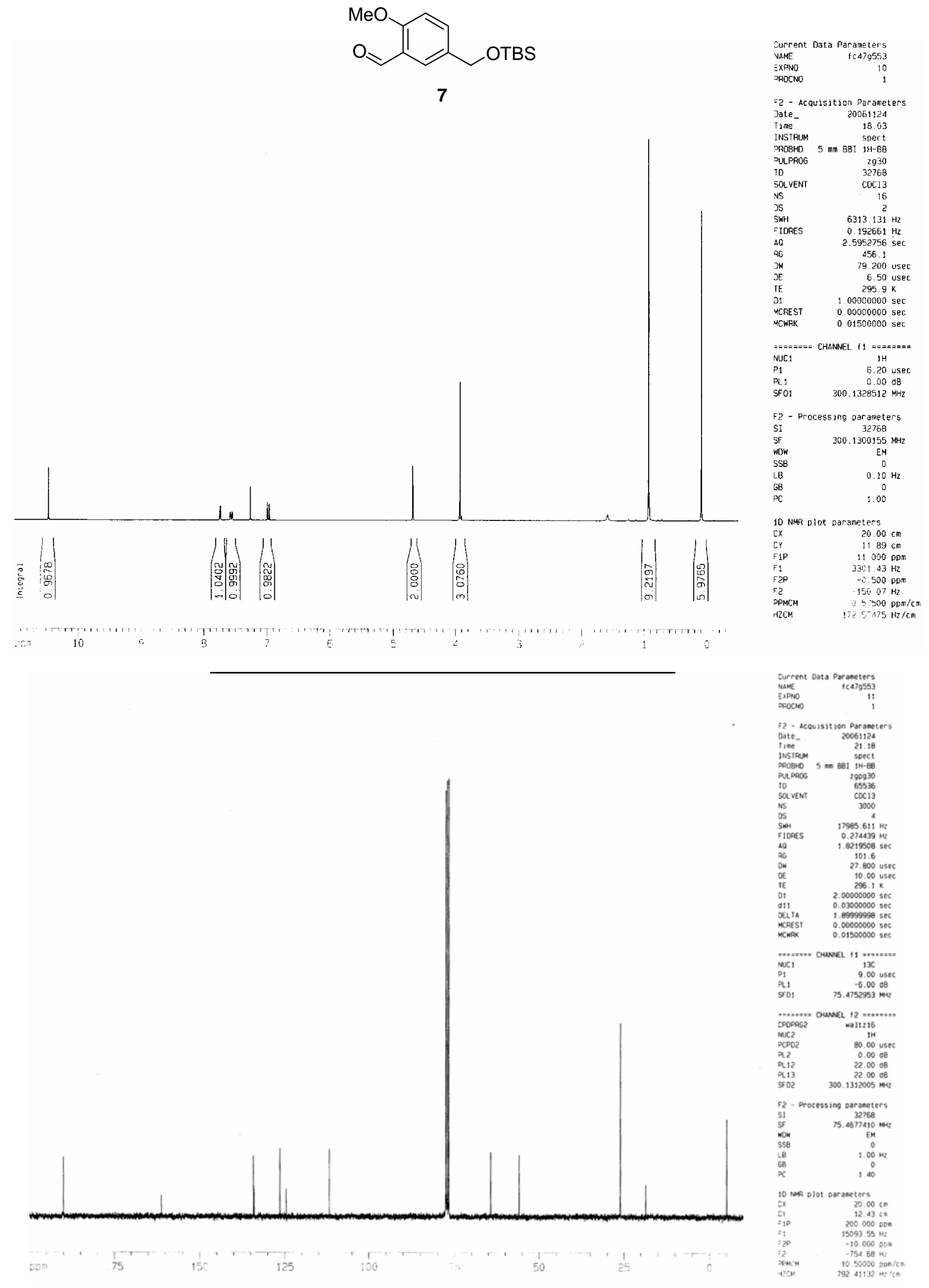




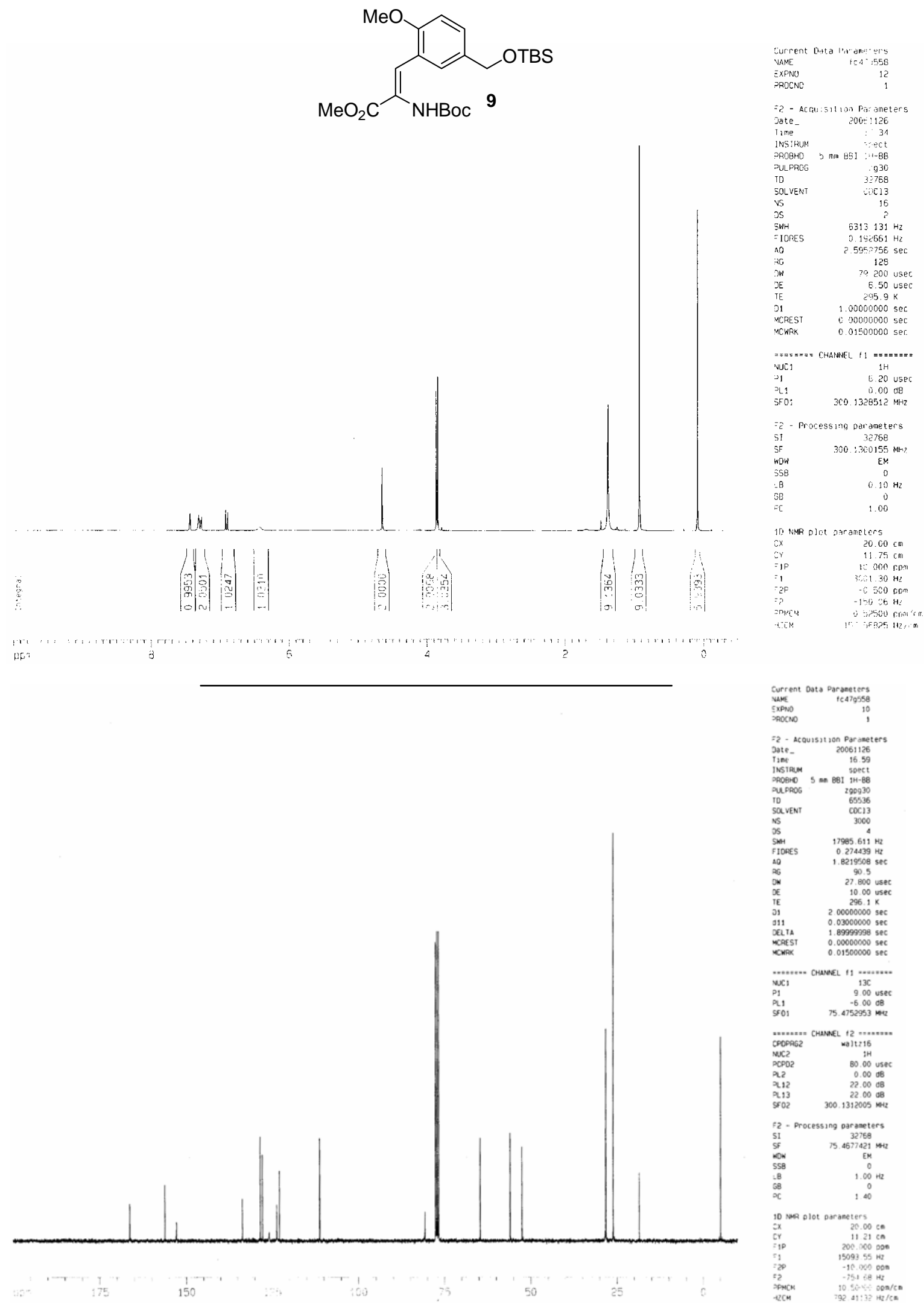



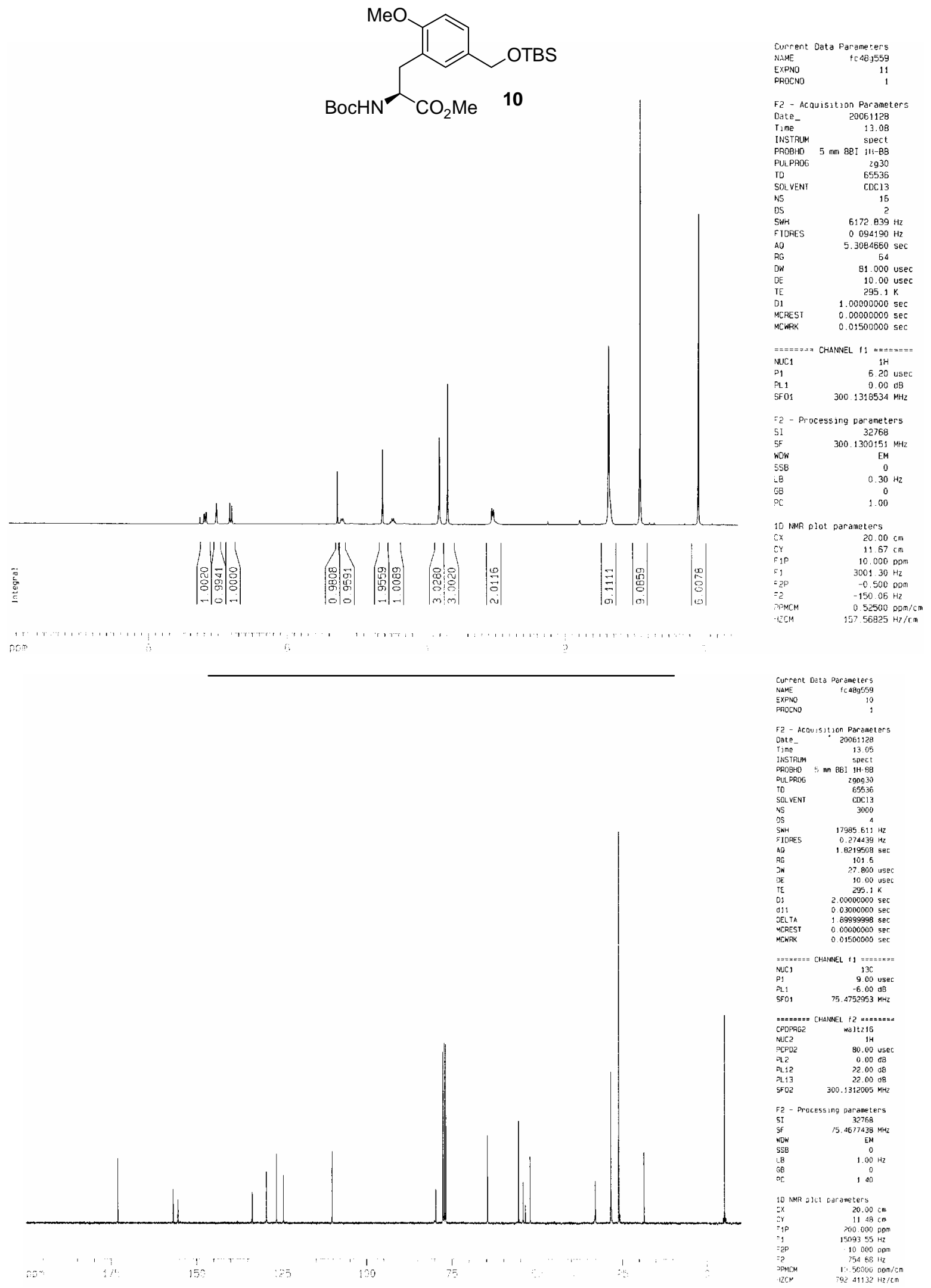


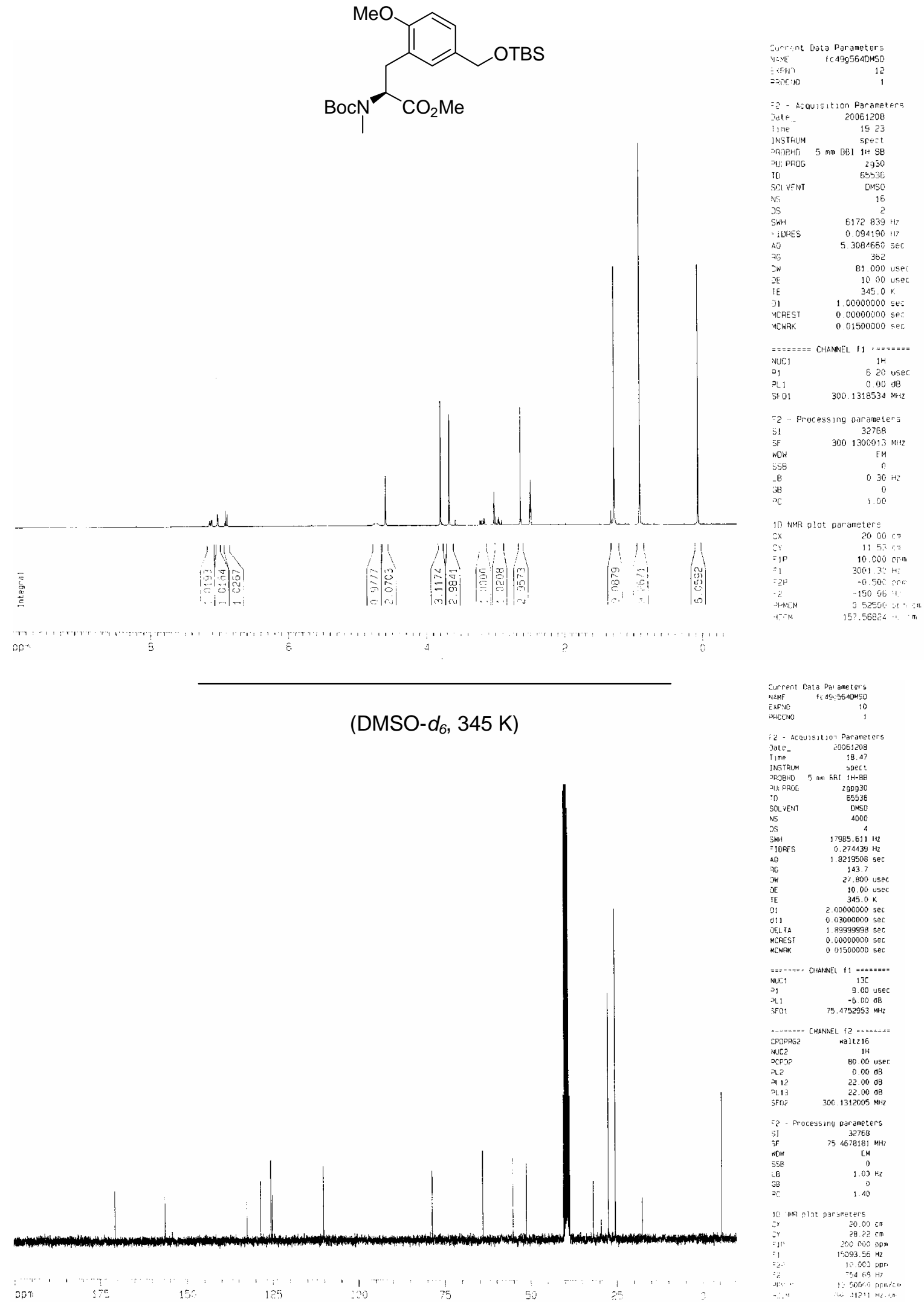




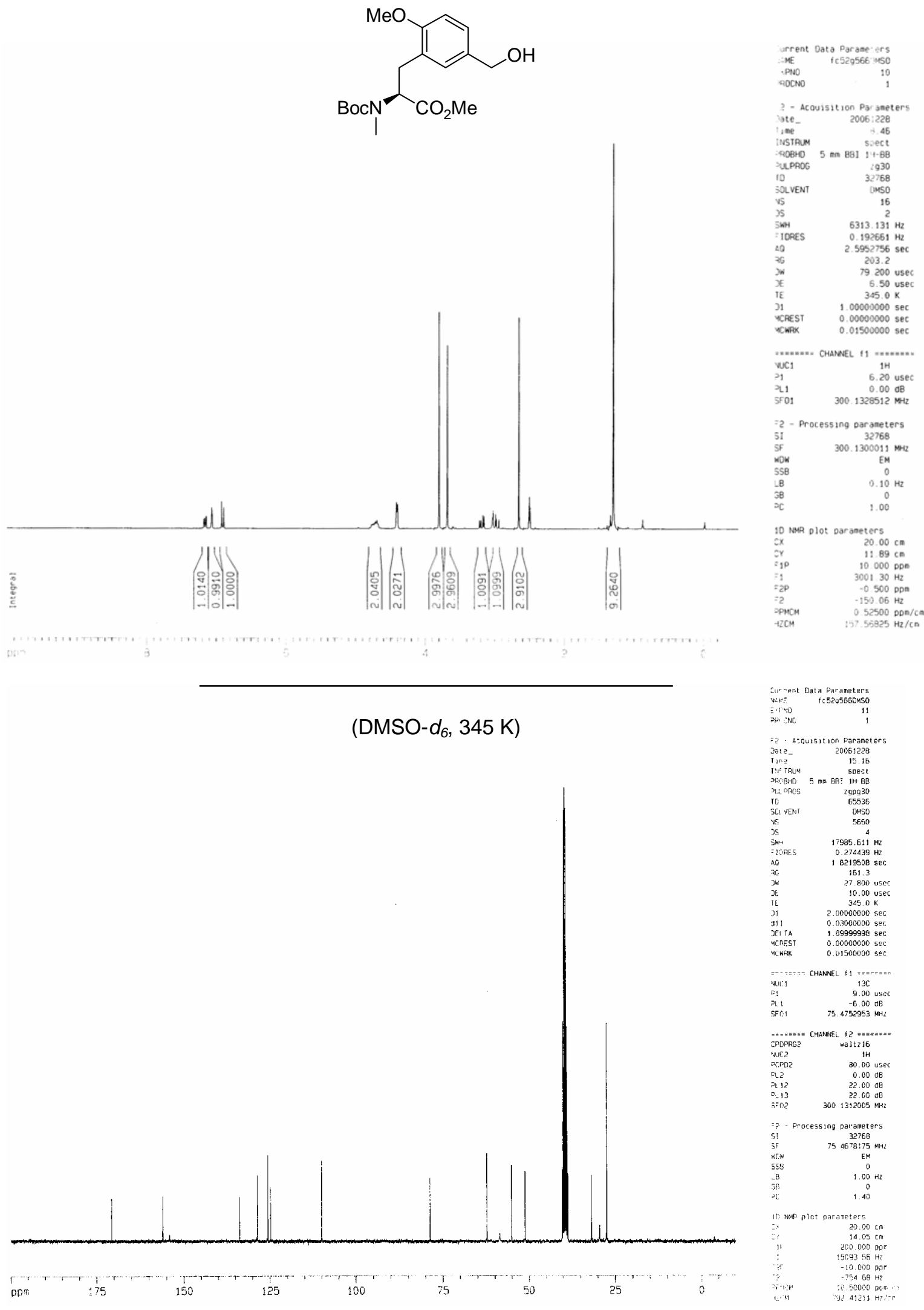




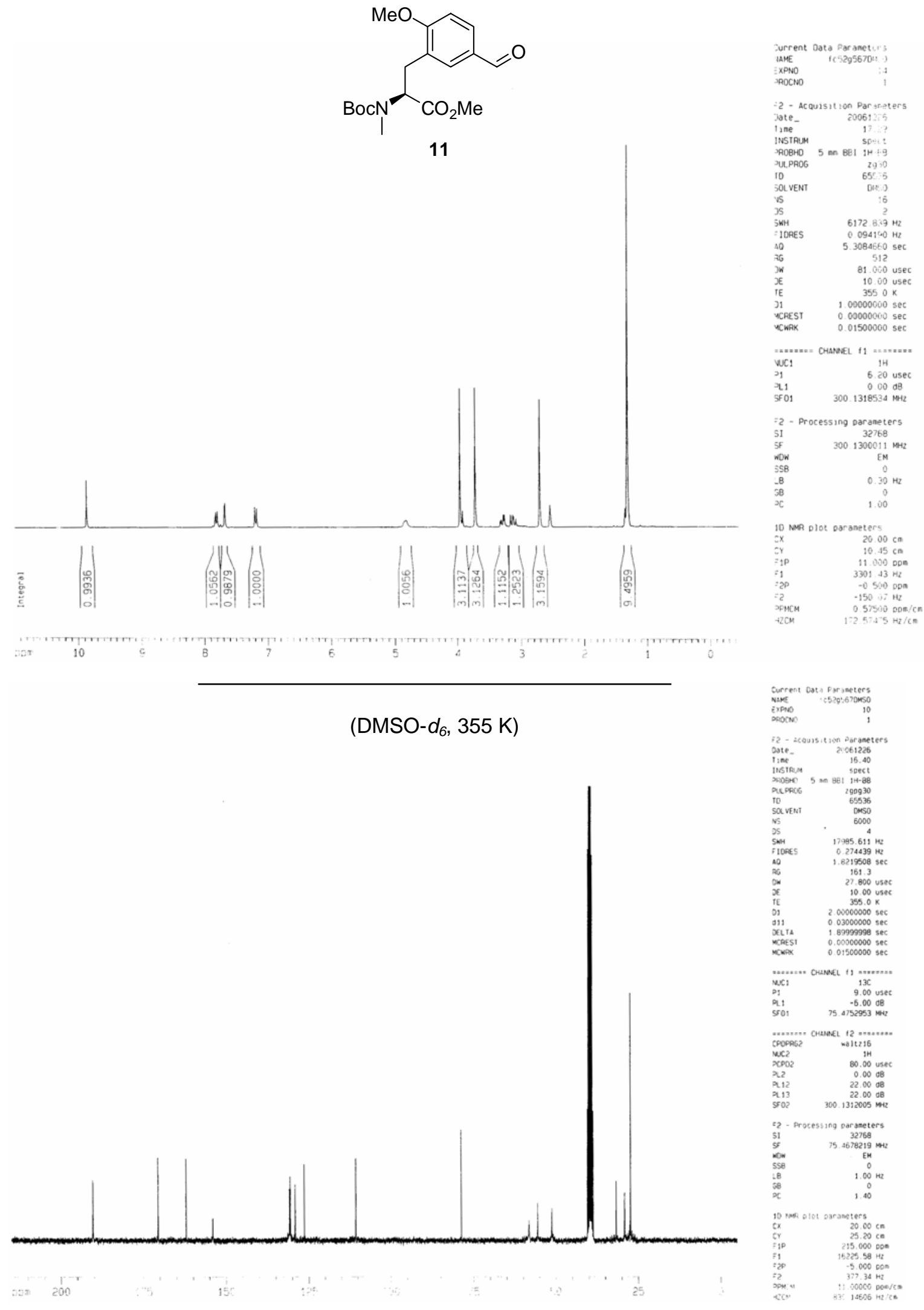




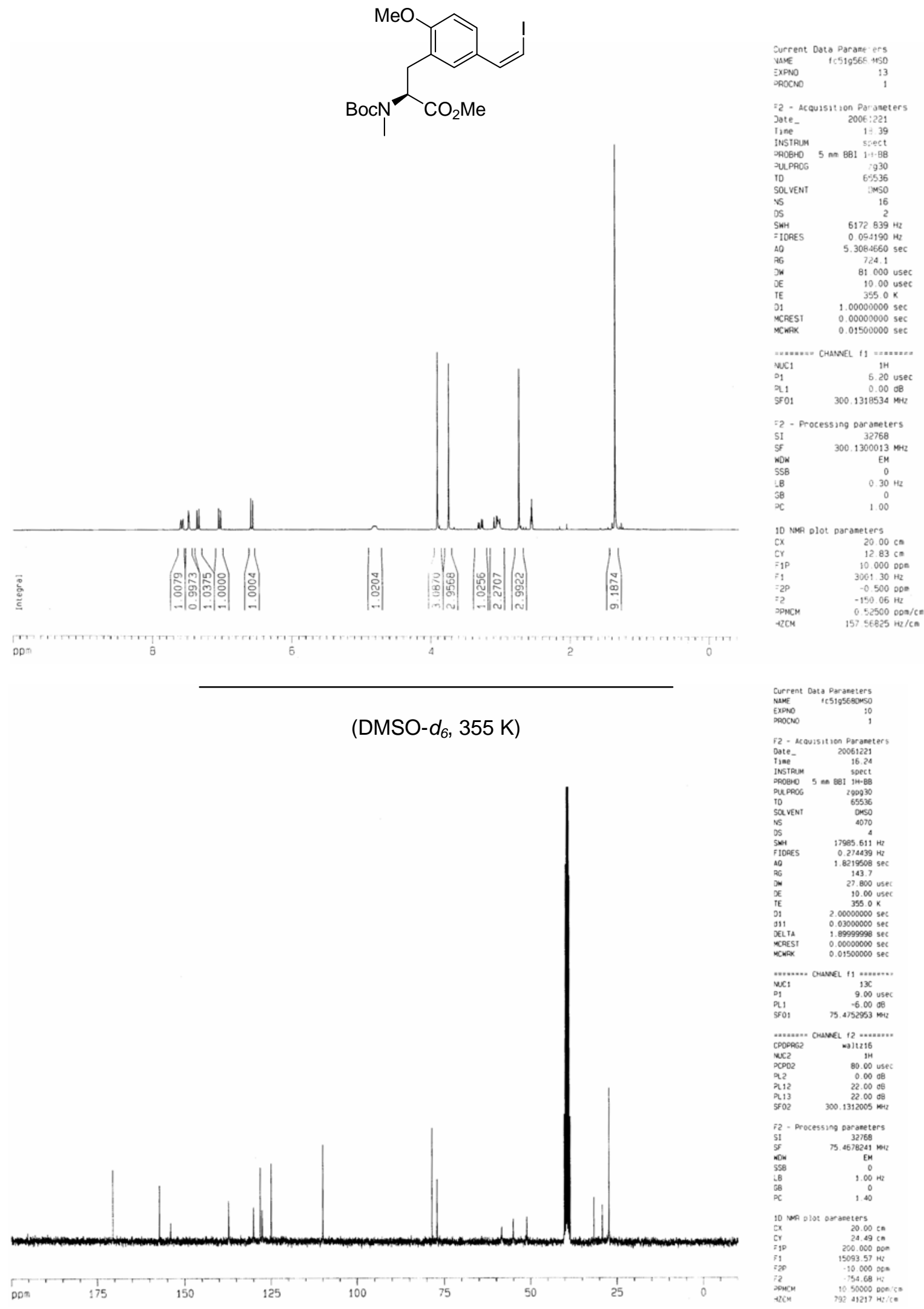




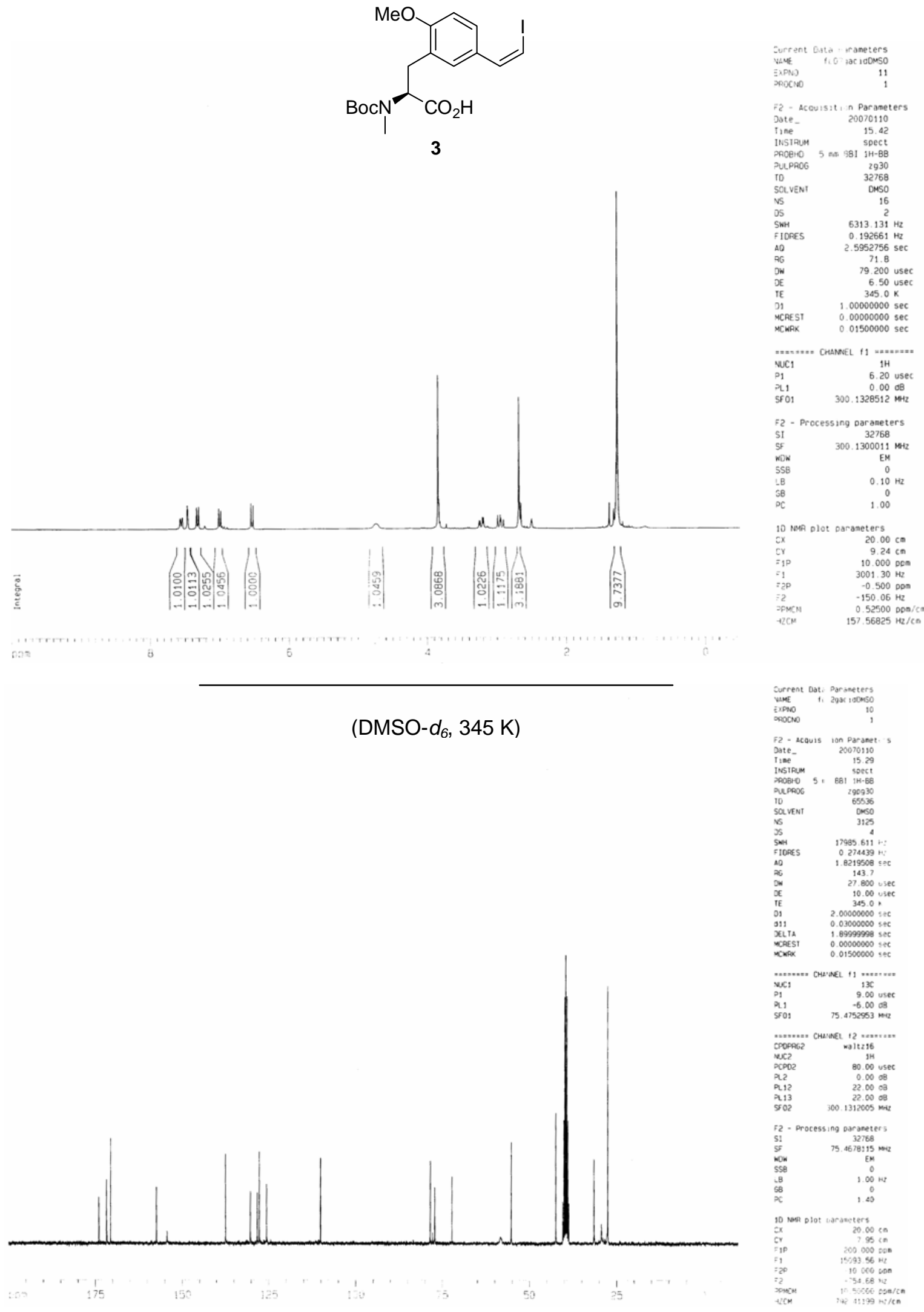




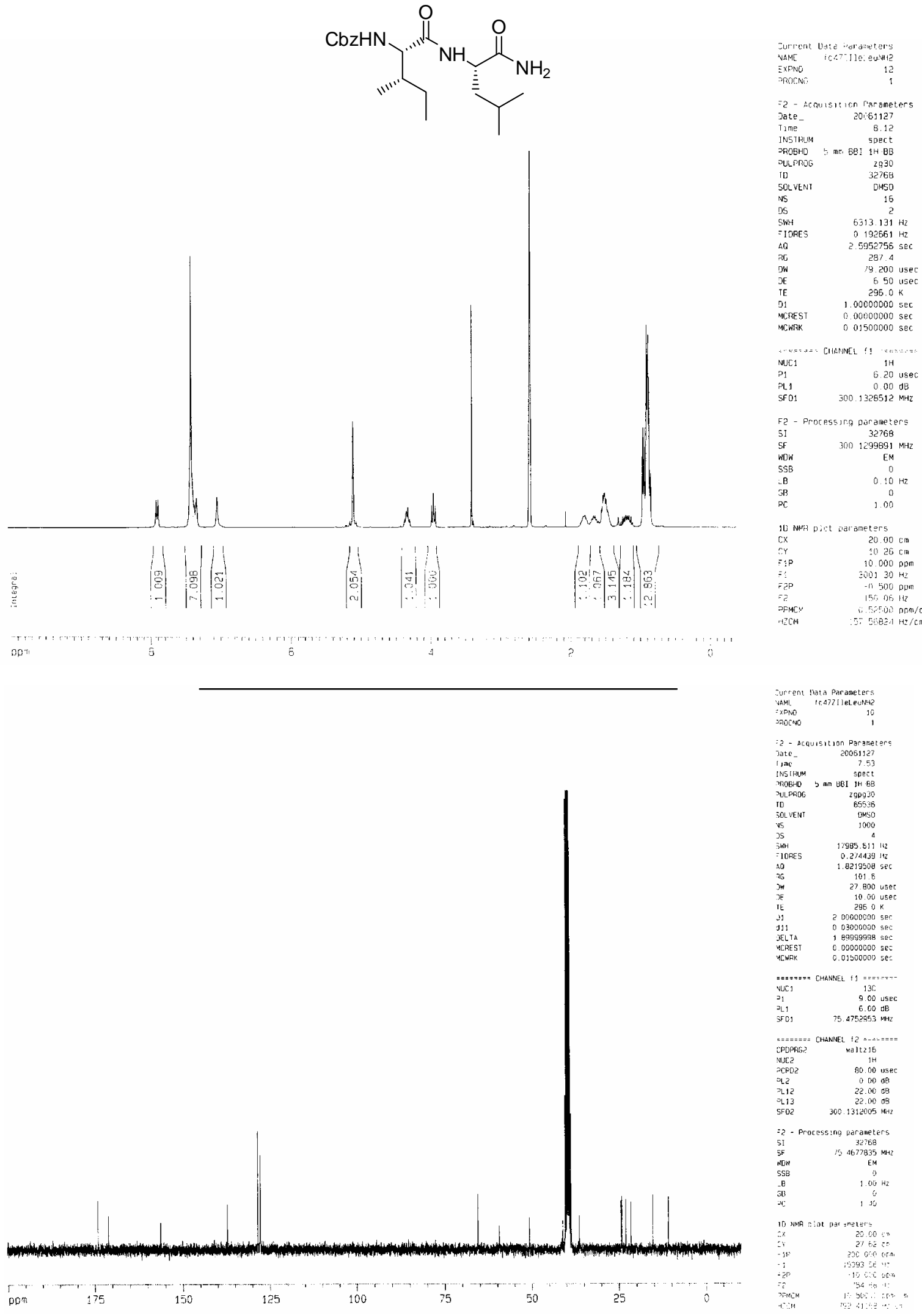




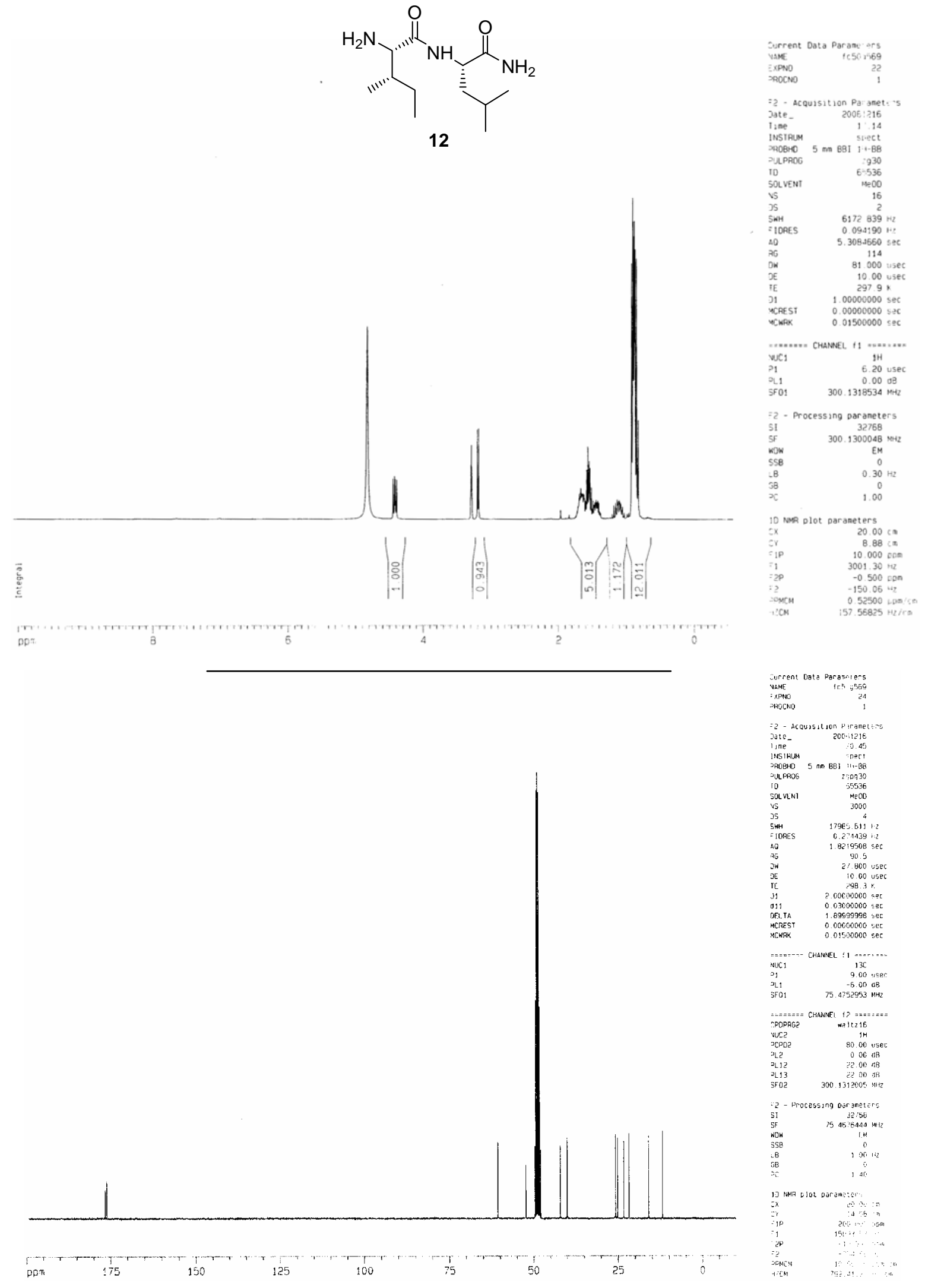




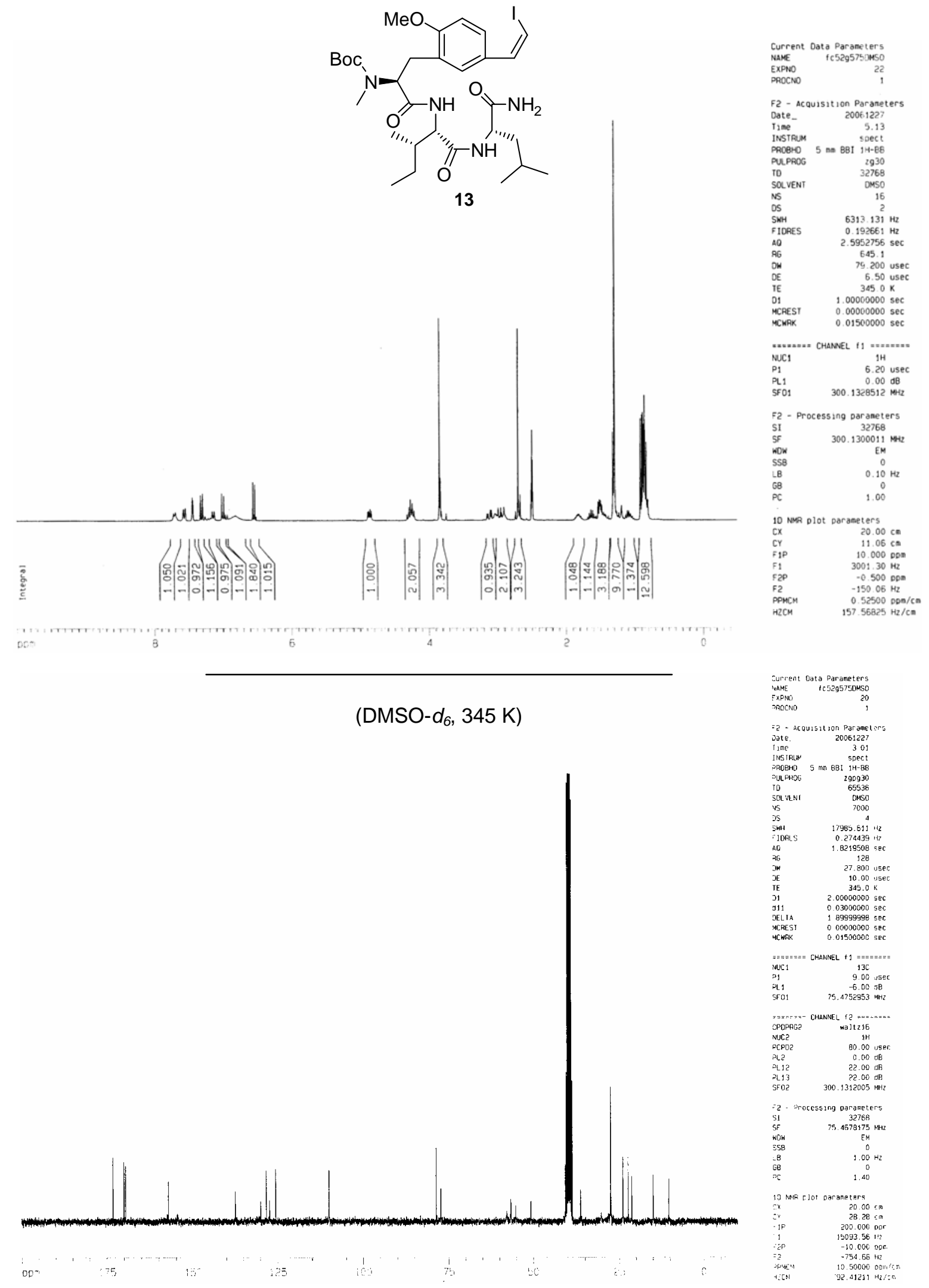




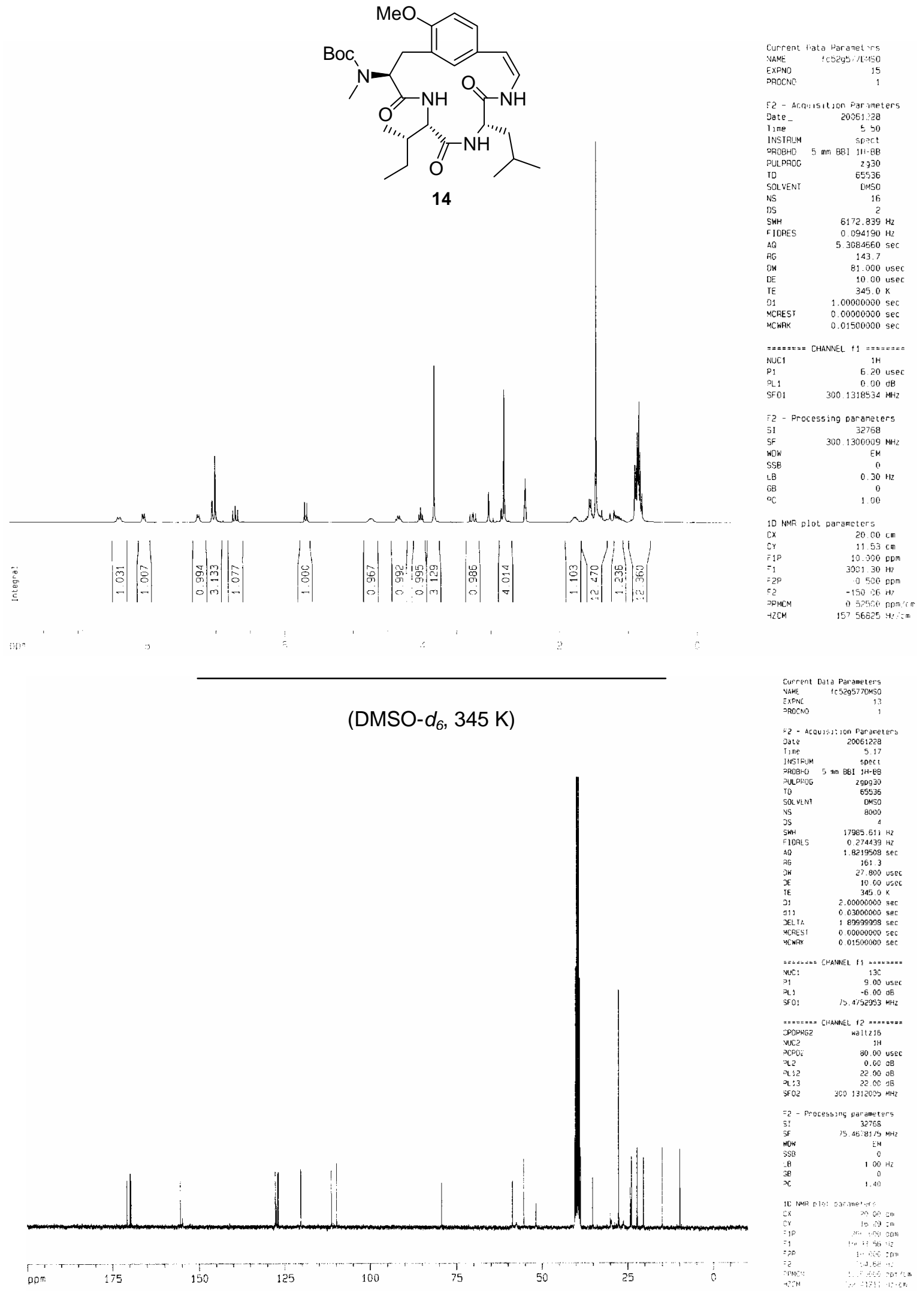



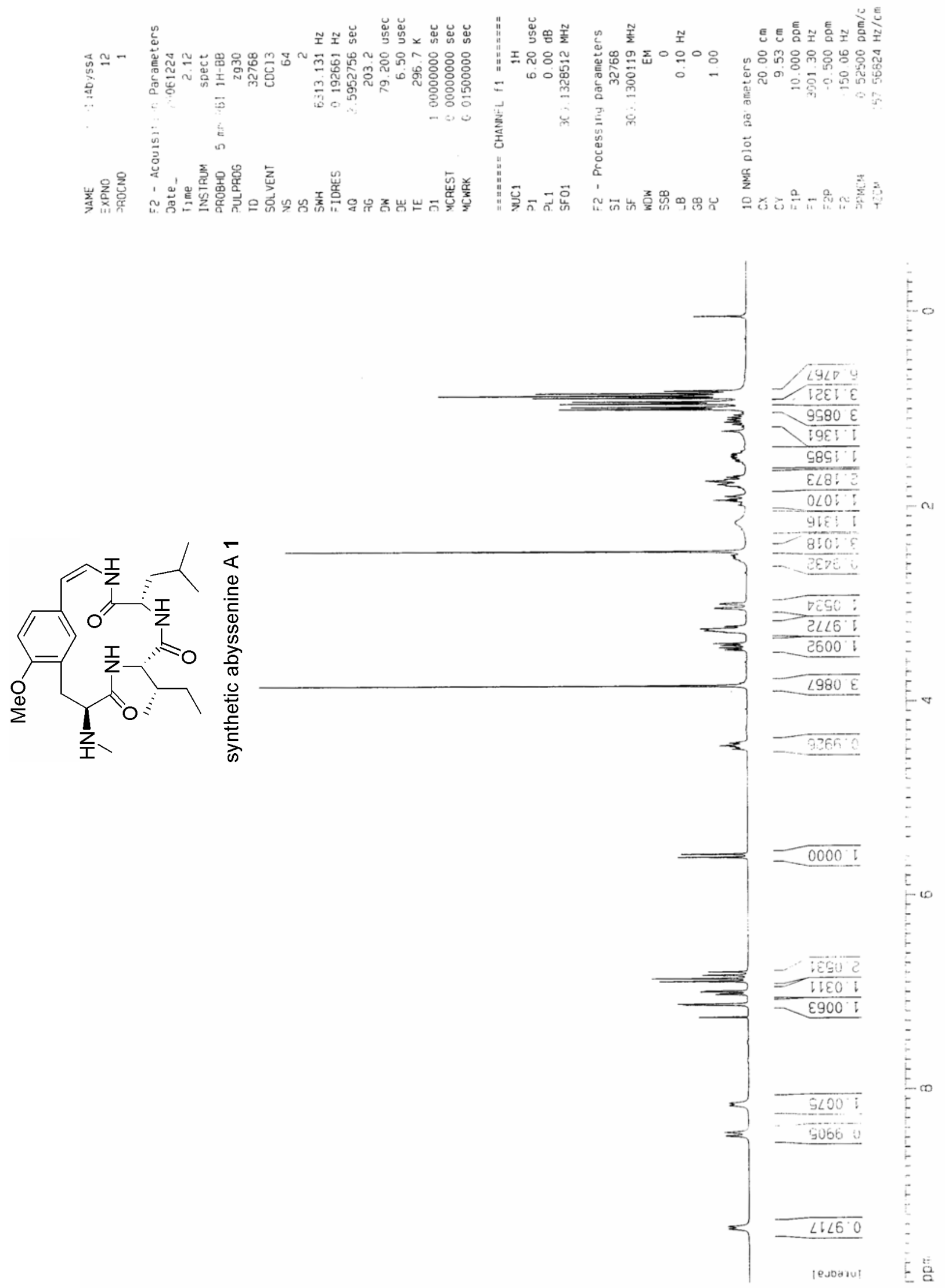


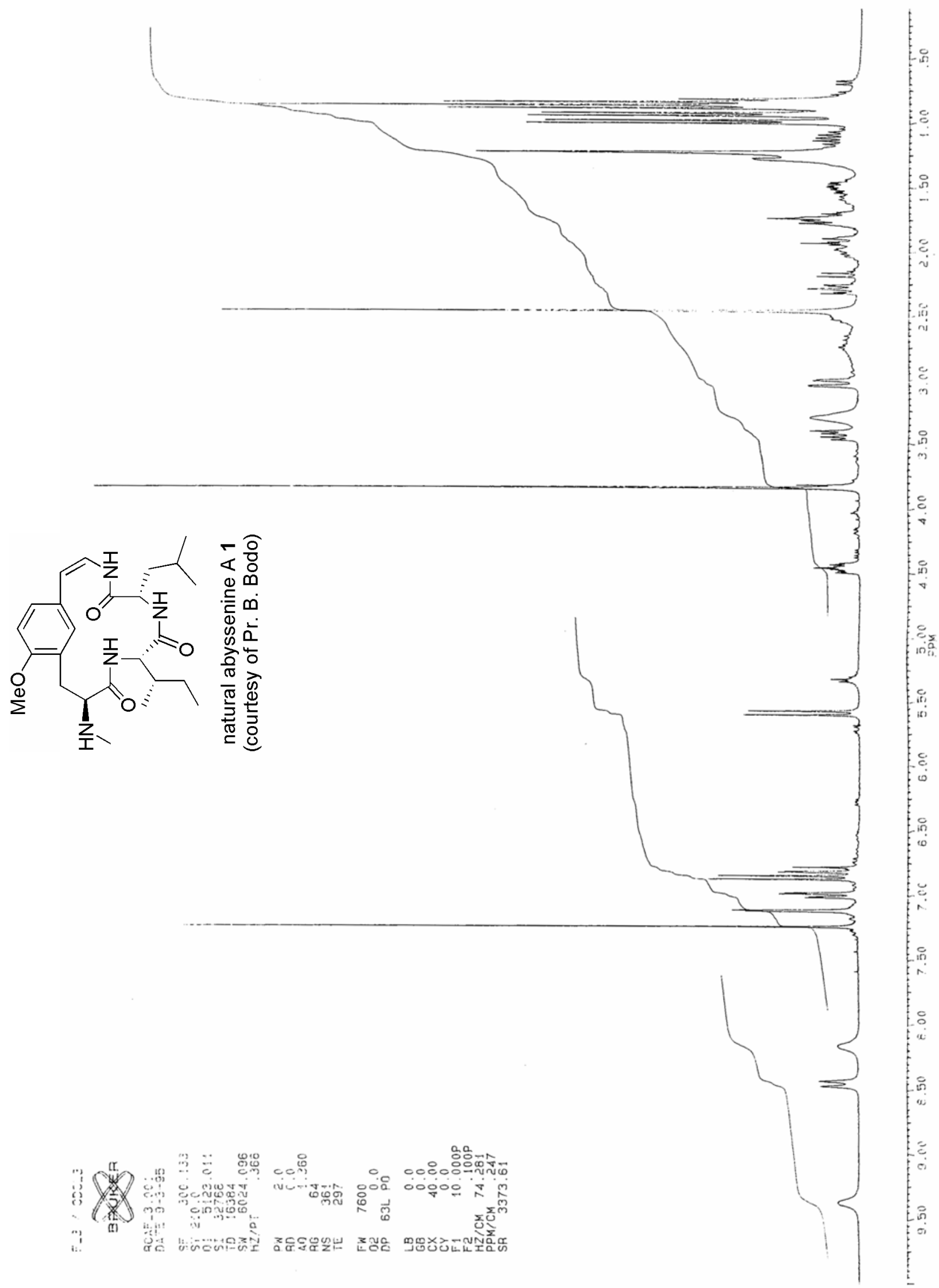



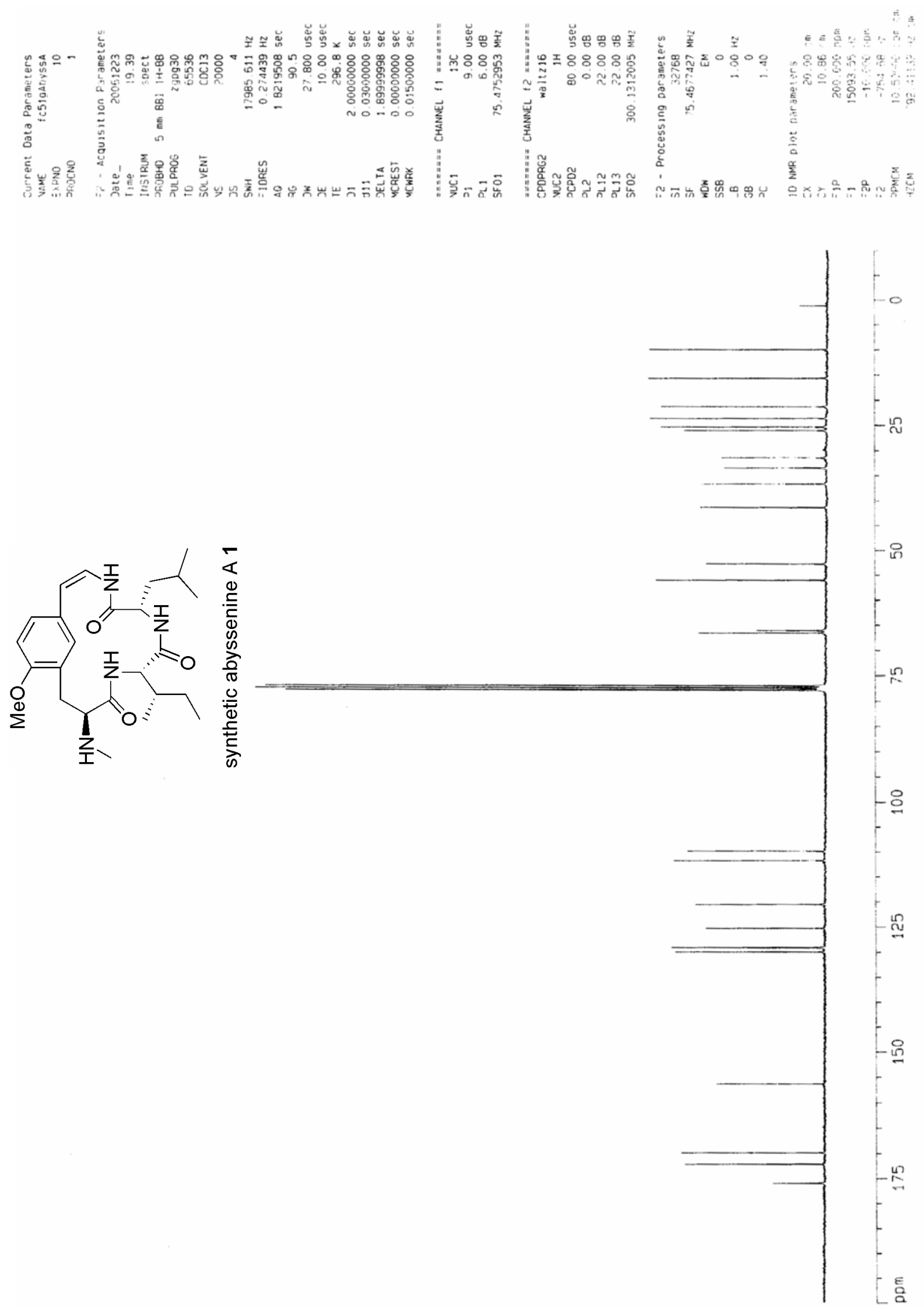
Supporting Information

\section{${ }^{1} \mathrm{H}-{ }^{1} \mathrm{H}$ COSY and ${ }^{1} \mathrm{H}-{ }^{13} \mathrm{C}$ HSQC NMR spectra of synthetic Abyssenine A}



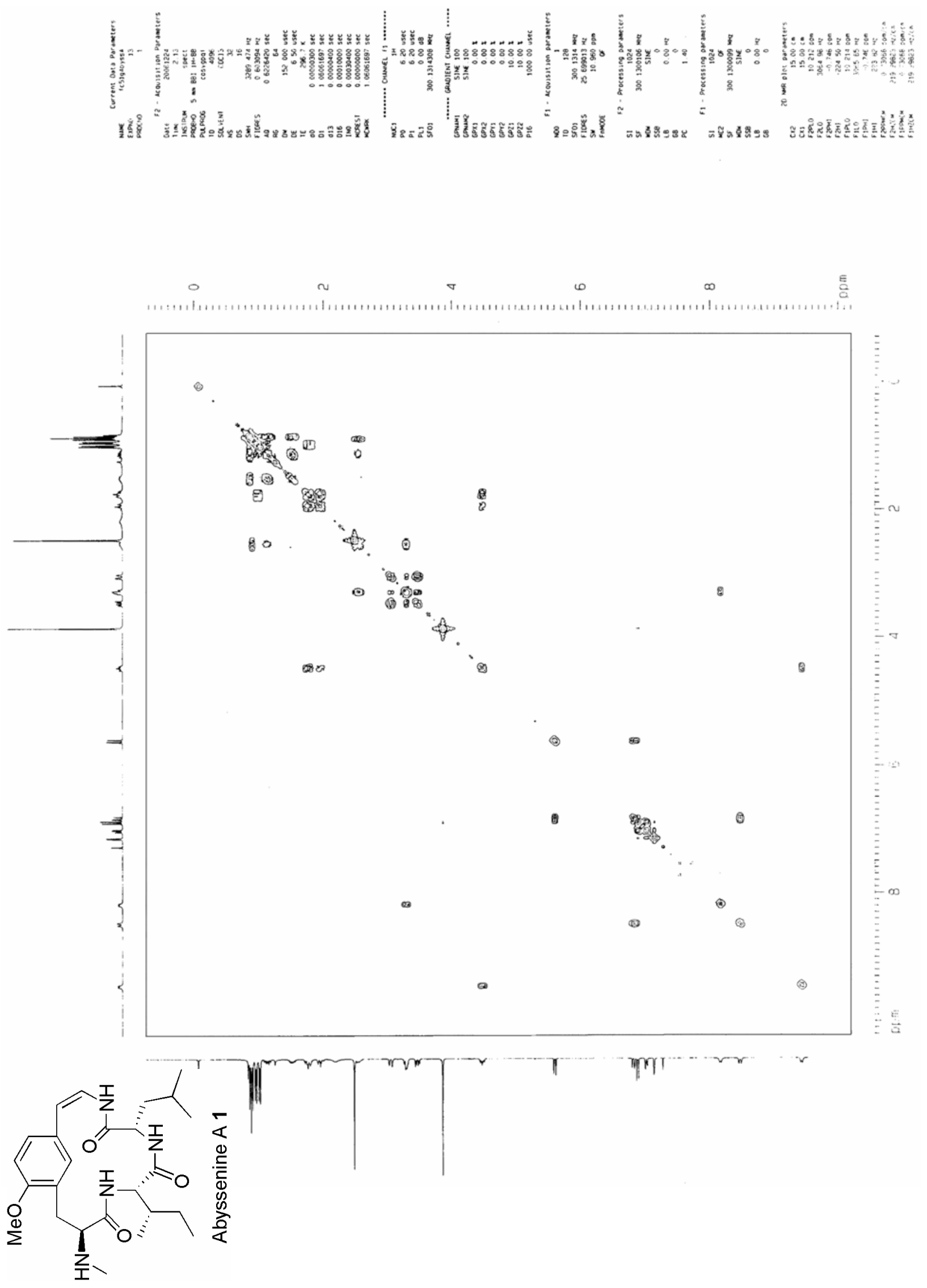


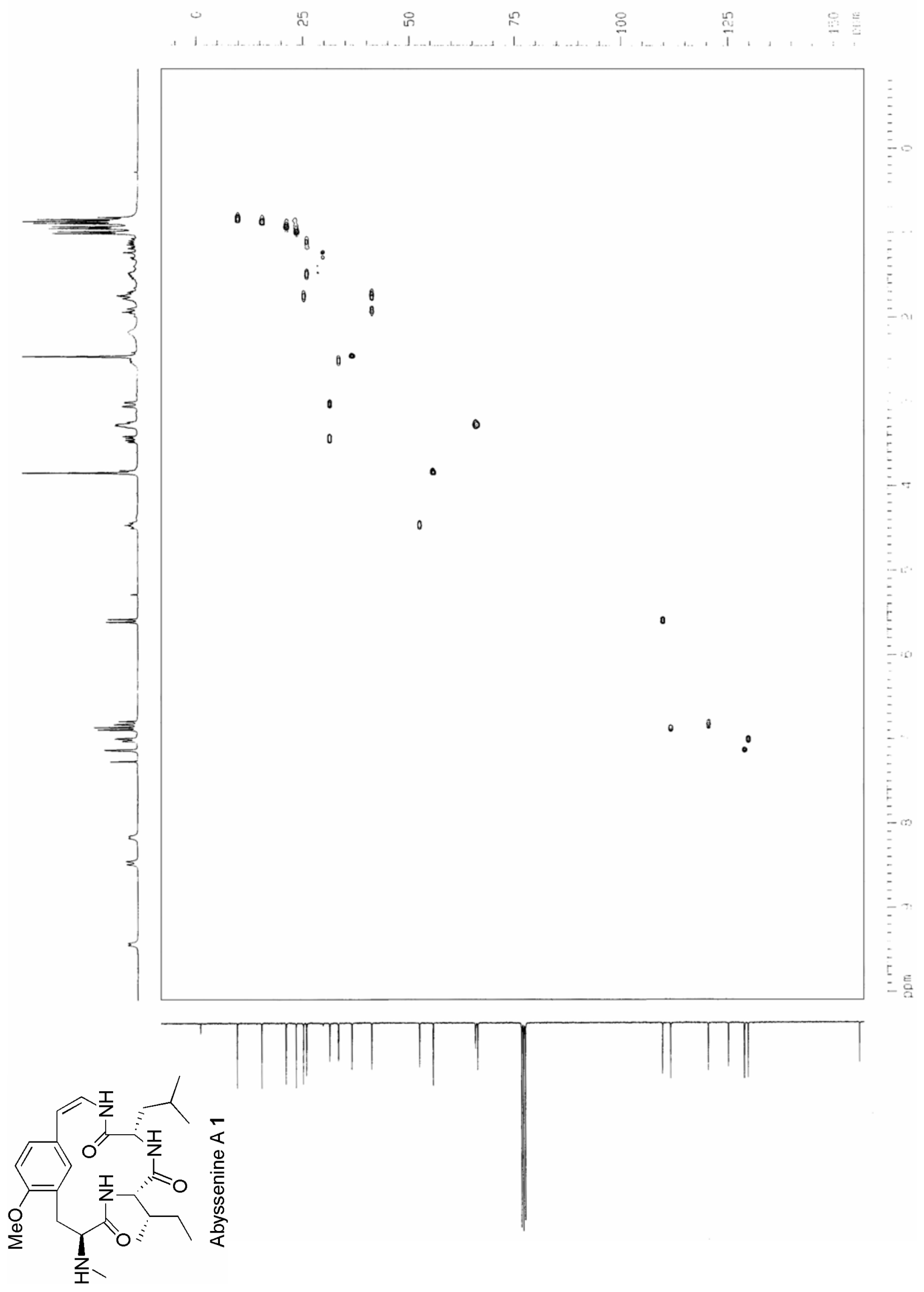

